\title{
Correction to: \\ Why and When Consumers Indulge in Smartphones: The Mental Association Between Smartphones and Fun, by Shen L, Wang L, and Zhang $X$. Cyberpsychology, Behavior, and Social Networking 2019: 22(6):381-387. DOI: 10.1089/cyber.2018.0646
}

N THE JUne 2019 ISSUE of Cyberpsychology, Behavior, and Social Networking (vol. 22, no. 6, pp. 381-387), the article

"'Why and When Consumers Indulge in Smartphones: The Mental Association Between Smartphones and Fun,'” by Liang Shen et al., contains an error in the column heads of Table 2 on page 383. The table compares hedonic and utilitarian data, but the column heads identified both sets of data as hedonic.

Study 2

\begin{tabular}{|c|c|c|c|}
\hline \multicolumn{2}{|c|}{ Smartphone } & \multicolumn{2}{|c|}{ Computer } \\
\hline $\begin{array}{l}\text { Hedonic-oriented } \\
\text { products }(\mathrm{N}=45)\end{array}$ & $\begin{array}{l}\text { Hedonic-oriented } \\
\text { products }(\mathrm{N}=47)\end{array}$ & $\begin{array}{l}\text { Hedonic-oriented } \\
\text { products }(\mathrm{N}=50)\end{array}$ & $\begin{array}{l}\text { Hedonic-oriented } \\
\text { products }(\mathrm{N}=54)\end{array}$ \\
\hline
\end{tabular}

They should read:

Study 2

\begin{tabular}{|c|c|c|c|c|}
\hline \multirow[b]{2}{*}{$\begin{array}{l}\text { Dependent } \\
\text { variables }\end{array}$} & \multicolumn{2}{|c|}{ Smartphone } & \multicolumn{2}{|c|}{ Computer } \\
\hline & $\begin{array}{l}\text { Hedonic-oriented } \\
\text { products }(\mathrm{N}=45)\end{array}$ & $\begin{array}{l}\text { Utilitarian-oriented } \\
\text { products }(\mathrm{N}=47)\end{array}$ & $\begin{array}{l}\text { Hedonic-oriented } \\
\text { products }(\mathrm{N}=50)\end{array}$ & $\begin{array}{l}\text { Utilitarian-oriented } \\
\text { products }(\mathrm{N}=54)\end{array}$ \\
\hline
\end{tabular}

The online version of the article has been corrected to reflect these changes.

The authors wish to apologize for the error. 